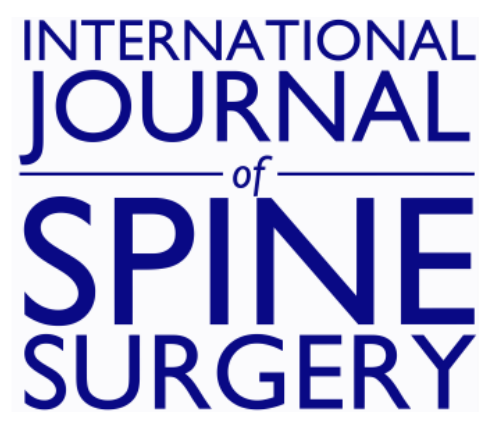

\title{
Outcomes of Negative Pressure Wound Therapies in the Management of Spine Surgical Site Wound Infections
}

SWAMY KURRA, AYMEN RASHID, HENAKU YIRENKYI, PATRICK CASTLE and WILLIAM F. LAVELLE

Int J Spine Surg 2020, 14 (5) 772-777

doi: https://doi.org/10.14444/7110

http://ijssurgery.com/content/14/5/772

This information is current as of April 26, 2023.

Email Alerts Receive free email-alerts when new articles cite this article. Sign up at:

http://ijssurgery.com/alerts

The International Journal of Spine Surgery

2397 Waterbury Circle, Suite 1,

Aurora, IL 60504, Phone: +1-630-375-1432 


\title{
Outcomes of Negative Pressure Wound Therapies in the Management of Spine Surgical Site Wound Infections
}

\author{
SWAMY KURRA, MBBS, ${ }^{1}$ AYMEN RASHID, MD ${ }^{1}$ HENAKU YIRENKYI, MD, ${ }^{1,2}$ PATRICK CASTLE, BS, ${ }^{1}$ \\ WILLIAM F. LAVELLE, MD ${ }^{1}$ \\ ${ }^{I}$ Department of Orthopaedic Surgery, SUNY Upstate Medical University, Syracuse, New York, ${ }^{2}$ OrthoAtlanta and Sports Medicine Specialists, Stockbridge, \\ Georgia
}

\begin{abstract}
Background: Many studies report benefits using negative pressure wound therapy (NPWT) in surgical site infections (SSIs). We measured and compared efficacy and complications associated with NPWT for traditional versus suprafascial vacuum-assisted closures (VACs).

Methods: This is a retrospective chart review of consecutive SSIs managed with negative wound therapy after spinal procedures between 2012 and 2015 from a single, academic center. Patients were collected through International Classification of Diseases, Ninth Revision, procedure codes. Inclusion criteria were patients with spine SSIs managed by irrigation and debridement with a VAC device; infection occurring after spinal surgeries; and age over 18 . A total of 23 consecutive patients met the criteria. We reviewed demographic data, surgical data, infectious disease data, discharge summaries, and postoperative follow-up charts. We compared and analyzed demographics, duration of VAC therapy, and reoperation rates between VAC groups. Statistical analysis was completed using analysis of variance and $\chi^{2}$ tests; $P$ $\leq 0.05$ was considered statistically significant.

Results: A total of 7 patients had traditional VACs (Group 1), and 16 patients had suprafascial VACs (Group 2). Average blood loss and number of levels involved during index surgery were not statistically significant between groups. Locations of infection occurrence were cervical spine $=3$, thoracic spine $=1$, and lumbar spine $=19$. Reoperation rate after initial wound VAC placement was $34 \%$, with rates significantly higher for Group $1(71 \%)$ than Group $2(16 \%), P$ $=0.02$. Average duration of wound therapy was longer in Group 1 ( 77 days) than Group 2 ( 33 days), $P=0.08$. Average number of operating room visits after initial wound VAC implantation were 0.7 for Group 1 and 0.3 for Group $2, P=$ 0.26 , before obtaining a clean wound closure.

Conclusions: Small sample size and retrospective nature were limitations. Negative pressure wound therapy may be useful for managing spinal infections, and suprafascial VAC had less time duration, lower risk of sponge fragment retainment, and fewer procedures to ultimately achieve wound closure.
\end{abstract}

Level of Evidence: 3.

Complications

Keywords: negative pressure wound therapy, vacuum-assisted closure (VAC), traditional vacuum-assisted closure, suprafascial vacuum-assisted closure, surgical site infections, spinal deformity surgery infections, complications

\section{INTRODUCTION}

Surgical site infections (SSIs) are potential complications of spine surgeries, with rates varying from $0.4 \%$ to $20 \%$ among published reports in the English literature. ${ }^{1,2}$ The current protocol treatment for SSIs for most surgeries is irrigation and debridement (I\&D) followed by appropriate antibiotic therapy. ${ }^{3}$ Spine surgeons have been using negative pressure wound therapy (NPWT) vacuum-assisted closure (traditional VAC) as an adjunct for managing SSIs after its benefits were reported in other areas. $^{3-5}$ Many studies have reported the benefits in the use of NPWT for SSIs, but only a few studies have concentrated on associated complications. $^{6}$

Surgeons use wound VAC devices in different ways. Some surgeons place the VAC sponge into the open wound bed (traditional VAC) and allow the device to facilitate tissue granulation until ultimate closure. Other surgeons believe that a bed of muscle is beneficial and place the VAC sponge over the patient's reapproximated paraspinal muscle (suprafascial VAC). To date, there are no studies that have reported or compared the differences between leaving the paraspinal muscles open or closed over the hardware. 
In this study, we measured and compared the complications associated with NPWT for both types of VACs and compared the efficacy between the VACs (traditional versus suprafascial).

\section{MATERIALS AND METHODS}

A retrospective chart review was conducted after our institutional review board (IRB) granted Exemption Category \#4 (exempt from IRB review). The patients for the study were collected through International Classification of Diseases, Ninth Revision, procedure codes (10180, postoperative wound infections; 97605 , wound VAC $\leq 50 \mathrm{~cm}^{2}$; and 97606, wound VAC $\geq 50 \mathrm{~cm}^{2}$ ) between 2012 and 2015. The inclusion criteria were patients with spine SSIs, managed by I\&D with wound VAC device implantation; infection occurring after spinal surgeries; and patients over age 18 at the time of surgery. We reviewed the patients' demographic data, surgical data, infectious disease data, discharge summaries, and postoperative follow-up charts from both the orthopaedic and infectious disease departments. Documentation included the patient's gender, age, premedical conditions, body mass index (BMI), indications for the index surgery, type of postoperative wound infections, preoperative and perioperative intravenous antibiotics, type of cultures (superficial and deep), and type of wound VAC therapy (traditional or suprafascial). Infectious disease charts were reviewed for the type of organism cultured and postoperative antibiotic therapy. Follow-up charts were reviewed for the number and types of subsequent invasive procedures, duration of wound VAC therapy, and spinal infections after completion of the treatment.

Our primary grouping variable was the type of wound VAC (traditional versus suprafascial) used. Patients who were managed initially with traditionally VAC were placed in Group 1, and those managed with suprafascial VAC were placed in Group 2. The demographics, duration of VAC therapy, reoperation rates, and average operations were compared between the 2 groups.

\section{Traditional and Suprafascial Wound VAC Procedures}

Patients with spine SSIs underwent I\&D under general anesthesia in the prone position. After the incision and purulent fluid drainage, deep and superficial cultures were taken. Aggressive debride- ment was performed until healthy bleeding tissue was seen, and then normal saline solution was used to irrigate the wound. In some procedures, vancomycin powder was placed inside the wound. After aggressive I\&D, surgeons either placed an appropriate wound-sized black VAC sponge (open-pored polyurethane sponge foam) deep in the wound (traditional VAC) or first reapproximated the paraspinal muscles and then placed the sized VAC sponge (black) over the reapproximated muscles (suprafascial VAC). A suction tube and sealant drape (sealed to a distance of $5 \mathrm{~cm}$ beyond the margins of the wound) were placed on top of the sponge, and the suction pressure was maintained at negative $125 \mathrm{mmHg}$ for both types of VAC placements. The wound VAC dressings were changed every 2 to 3 days, and therapy was continued until signs of wound closure were noticed.

\section{Statistical Methods}

Statistical analysis for the study was done by using IBM SPSS Statistics 22 software (IBM Corp, Armonk, NY). All collected variables were compared between these 2 groups. An analysis of variance (ANOVA) test was used to compare the continuous variables, and a $\chi^{2}$ test was used for categorical variables; $P \leq 0.05$ was considered statistically significant.

\section{RESULTS}

The study group was comprised of 23 consecutive patients with a mean age of 59 years (range $=25$ 84). With respect to gender, there were 10 males and 13 females. The average blood loss was $1282 \mathrm{~mL}$, and the average levels of surgery were 7 . Three infections occurred in the cervical spine, 1 in the thoracic spine, and the remaining in the lumbar regions $(n=19)$ of the spine. Index spinal procedures were performed to address scoliosis, degenerative scoliosis, L5 burst fracture, L1 burst fracture, and cervical pathologies. The indications for I\&D and VAC placements were a spinal draining wound, lumbar abscess, deep wound infection, wound infection with bacteremia with or without meningitis, and wound infection with cerebrospinal fluid leak. Seven patients $(30 \%)$ in the study had diabetes. Two patients had an incidental durotomy with wound infection. Incidental durotomy patients who had lumbar drain 
Table 1. Patient demographics.

\begin{tabular}{|c|c|c|c|c|}
\hline & Group 1: Traditional VAC & Group 2: Suprafascial VAC & Test & $P$ Value \\
\hline $\mathrm{n}$ & 7 & 16 & & \\
\hline Mean age, y & 52 & 69 & ANOVA & 0.08 \\
\hline Mean BMI & 31 & 32 & ANOVA & 0.76 \\
\hline Mean CCI & 1.7 & 3 & ANOVA & 0.05 \\
\hline Diabetes, \% & $29(\mathrm{n}=2)$ & $31(\mathrm{n}=5)$ & $\chi^{2}$ & 0.64 \\
\hline Surgery levels, average (range) & $7(2-16)$ & $7(2-18)$ & ÂNOVA & 1.00 \\
\hline Blood loss, average (range), mL & $1360(200-2300)$ & $1250(200-3000)$ & ANOVA & 0.81 \\
\hline
\end{tabular}

Abbreviations: ANOVA, analysis of variance; BMI, body mass index; CCI, Charlson Comorbidity Index; VAC, vacuum-assisted closure.

placement also received prophylactic antibiotics for meningitis.

All the patients in our study received preoperative intravenous prophylactic empirical antibiotics, except 2 patients who received antibiotics after cultures were taken. Every patient had a follow up of greater than 6 months. Methicillin sensitive (MSSA) and resistant Staphylococcus aureus (MRSA), Staphylococcus epidermis (SE), gram negative rods (Escherichia coli), Klebsiella pneumoniae, Enterococcus fecalis, and propyonibacterium were grown in cultures isolated during I\&D. Three patients had multiple microbial cultures, and 1 patient's cultures were negative. Once the growth cultures revealed the causative organism, all patients were placed on the Infectious Disease Department's recommended appropriate antibiotic therapy for at least 4 to 6 weeks.

There were 7 patients in Group 1 (traditional VAC) and 16 patients in Group 2 (suprafascial $\mathrm{VAC}$ ). The average blood loss and number of levels involved during the index surgery were not statistically significant between the groups. The average age of the patients was slightly higher in Group 2 (Table 1). The diabetes rate and average BMI were comparable between both groups. Charlson Comorbidity Index (CCI) was significantly higher in Group 2. The reoperation rate after the initial wound VAC placement was $34 \%$. In Group 1, 5 out of 7 patients required reoperations. In Group 2, 3 out of the 16 patients had reoperations. The reoperation rate was significantly higher for traditional VAC $(71 \%)$ than suprafascial VAC $(16 \%), P$ $=0.02$. The average duration of wound therapy was longer in the traditional VAC group at 77 days (range $=7-235$ days) than the suprafascial VAC group at 33 days (range $=3-116$ days), $P=0.08$ (Table 2). The average number of operating room visits after initial I\&D and wound VAC implantation was 0.7 (range $=0-1$ ) in the traditional VAC group and $0.3($ range $=0-2)$ in the suprafascial VAC group $(P=0.26)$, before obtaining a clean wound closure. All patients achieved clean wound closure without removal of instrumentation. One patient in the suprafascial VAC group had a new infection after the closure of the wound, and none were reported in the traditional VAC group.

In the study, 23 patients overall received 32 I\&D procedures with adjunct VAC implantations. Nine patients had recurrent persistent spine infections. Six of the cultures $(26 \%)$, taken at the time of the initial I\&D procedure, were positive for MSSA, $0.4 \%(n=1)$ for MRSA, and $21 \%(n=5)$ for SE. Eighty percent of the MSSA-positive patients $(n=4)$ managed with traditional VAC revisited the operating room for persistent infections. In the suprafascial group, 1 patient $(33 \%)$ revisited the operating room. This difference was not statistically different between the groups, $P=0.28$. All the revisited MSSA patients in both traditional and suprafascial VAC groups were successfully managed with suprafascial VAC therapy without any operating room revisits before the closure of the wound. One MRSA-positive patient was managed initially with traditional VAC but revisited the operating room for a persistent infection which was successfully treated with suprafascial VAC. For SE-positive patients, from the traditional VAC group, 1 positive patient was reoperated for hardware failure and S1 vertebrae lytic destruction and fracture, and in the

Table 2. Patient outcomes by wound VAC type.

\begin{tabular}{|c|c|c|c|c|}
\hline & Group 1: Traditional VAC & Group 2: Suprafascial VAC & Test & $P$ Value \\
\hline Revision surgeries for persisted infections & $\mathrm{Yes}=5, \mathrm{No}=2$ & $\mathrm{Yes}=3, \mathrm{No}=13$ & $\chi^{2}$ & 0.026 \\
\hline Duration of wound VAC therapy, $d$ & $77($ range $=7-235)$ & $33($ range $=3-116)$ & ANOVA & 0.08 \\
\hline Reoperations, mean (range) & $0.7(0-1)$ & $0.3(1-2)$ & ANOVA & 0.27 \\
\hline
\end{tabular}

Abbreviations: ANOVA, analysis of variance; VAC, vacuum-assisted closure. 
suprafascial VAC group, 1 patient, who had a history of multiple SE infections, revisited the operating room for a persistent SE infection.

\section{DISCUSSION}

Spinal wound infections are one of the complications of spinal deformity surgeries. These complications are a burden for the patients by increasing the number of hospital visits, antimicrobial resistance, antibiotic use, and length of hospital stays. Most authors recommend I\&D as the treatment of choice combined with an antibiotic regime for these spine SSIs. The average operating room visits ranged from 2.7 to 4.7 times before complete closure of the wound for spinal wound infections. ${ }^{7,8}$ Wound VAC as an adjunct with aggressive I\&D and an appropriate follow-up antibiotic regime have been proven to decrease the number of operating room visits before the closure of the wound and effective for the treatment of spinal wound infections. ${ }^{3,5,9,10}$

Studies have reported patient-related factors, such as diabetes, smoking, alcohol abuse, malnutrition, drug abuse, malignancy, morbid obesity, radiation before surgery, and cardiovascular problems, as risk factors for postoperative infections, and these risk factors can potentially be responsible for the resistance of infection for VAC therapy. ${ }^{1,11}$ Along with patient-related risk factors, surgeryrelated factors, such as number of levels of fusion, revision surgery, and staging have been correlated with higher infection rates. ${ }^{3,11}$ In this study, $30 \%$ of the patients had diabetes, and the rate was no different between groups. Furthermore, the CCI was significantly higher in the suprafascial VAC group.

The literature maintains that a wound VAC works on the concept of negative pressure in the wound, but this concept remains unclear. ${ }^{6}$ It appears that there are multiple methods that work in unison. Negative pressure improves blood flow and angiogenesis rate and reduces local edema, which helps in the formation of granulation tissue. ${ }^{1}$ Microbes are removed from the wound in 2 ways: more blood flow leads to a greater antibiotics supply to the wound, and the negative pressure physically removes the bacteria. ${ }^{12}$ In addition, it is an effective way to reduce dead space, which prevents contamination and desiccation of the wound. ${ }^{13}$ The current treatment protocol for using wound VAC involves placement of the sponge after an initial I\&D. The negative pressure system is then used until either the wound can be closed in a delayed primary fashion or by secondary intention. Additional trips to the operating room for further debridements may also occur.

The average duration for traditional VAC therapy was 77 days (range $=7-235$ days) in our study compared with 3 to 186 days reported in other studies. ${ }^{1}$ For suprafascial VAC patients in our study, the duration of the therapy was 33 days (range $=3-116$ days), which was significantly lower than traditional VAC therapy.

Reported complications related to the use of wound VAC therapy, while rare, are limited. A review done by Ousey et $\mathrm{al}^{1}$ in 2013 found 4 studies that reported complications. Jones et $\mathrm{al}^{6}$ noticed bleeding complications related to VAC devices where 2 patients had reoperations for persistent infections: 1 patient required a skin graft for nonhealing granulation tissue, and the other patient died of delayed complications related to intraoperative blood loss via the VAC system after refusal of a blood transfusion on religious grounds. Labler et $\mathrm{al}^{13}$ reported on 13 patients with infected spinal hardware treated with traditional VAC. The spinal hardware was replaced in 7 patients and removed in 5. Two wounds required muscle flaps for final closure. ${ }^{13}$ In our study, no bleeding complications were reported in either group. Other complications reported in the literature include the possibility of minimal bleeding at dressing changes, formation of fistulas, cerebrospinal fluid leak, and local wound rash, ${ }^{1,6}$ yet none of these complications occurred in our study.

Retained fragments of the sponge in the wound can cause a persistent infection with traditional VAC. ${ }^{14}$ In suprafascial VAC treatment, the sponge is placed on the top of the reapproximated muscles, so the risk of sponge fragment retainment is very low. Late infections after complete closure of wound have been reported outside of the spine literature. ${ }^{6}$ In our study, 1 late infection was reported in a patient who was managed by suprafascial VAC after the complete closure of the wound, but none were reported in the traditional VAC group.

Ploumis et $\mathrm{al}^{11}$ reported that, after the initial VAC was placed, there was an average of 1.4 procedures until the closure of the wound. Mehbod et $\mathrm{al}^{3}{ }^{3}$ in their study, reported the average number of visits to the operating room for patients was 2.2 (23 ) after the placement of a VAC device for persistent 
infections. In our study, the average number of procedures was 0.7 (range $=0-1$ ) for traditional VAC and 0.3 (range $=0-2$ ) for suprafascial VAC. There was no statistical difference between the 2 VAC procedures, possibly due to our small sample size.

Multiple studies have shown that the use of a negative pressure system reduces the time for wound closure, number of operations, and the duration of hospital stays. However, there does not appear to be any literature studying the difference whether reapproximating the paraspinal muscles (suprafascial VAC) changes the outcomes. In our study, the traditional VAC therapy patients had a longer duration of therapy than the suprafascial VAC therapy with a statistical difference of $P=0.08$. Additionally, reoperation rates were also significantly higher for the traditional VAC than suprafascial VAC. One reason, to explain these possible differences, may be the decreased dead space that the wound has to be closed over with granulation tissue. Another explanation may be the improved biology with the larger soft tissue envelope around the wound VAC, which allows for increased blood flow and angiogenesis.

The small sample size and retrospective nature are limitations of our study. Another limitation is that all suprafascial VACs were performed by 1 surgeon. A multicenter study with a larger number of patients is needed to support our findings.

\section{CONCLUSIONS}

In conclusion, negative pressure therapy may be a useful tool for managing spinal infections. In addition, we have been able to show that, if possible, reapproximating the paraspinal muscles (suprafascial VAC) allows for less time duration, lower risk of sponge fragment retainment, and fewer procedures to ultimately achieve closure of the wound.

\section{REFERENCES}

1. Ousey KJ, Atkinson RA, Williamson JB, Lui S. Negative pressure wound therapy (NPWT) for spinal wounds: a systematic review. Spine J. 2013;13(10):1393-1405. doi:10. 1016/j.spinee.2013.06.040

2. Argenta LC, Morykwas MJ. Vacuum-assisted closure: a new method for wound control and treatment: clinical experience. Ann Plast Surg. 1997;38(6):563-576; discussion 577. doi:10.1097/00000637-199706000-00002

3. Mehbod AA, Ogilvie JW, Pinto MR, et al. Postoperative deep wound infections in adults after spinal fusion: manage- ment with vacuum-assisted wound closure. J Spinal Disord Tech. 2005;18(1):14-17. doi:10.1097/01.bsd.0000133493.32503. d3

4. van Rhee MA, de Klerk LW, Varhaar JA. Vacuumassisted wound closure of deep infections after instrumented spinal fusion in six children with neuromuscular scoliosis. Spine J. 2007;7(5):596-600. doi:10.1016/j.spinee.2006.09.002

5. Zehnder SW, Place HM. Vacuum-assisted wound closure in postoperative spinal wound infection. Orthopedics. 2007;30(4):267-272.

6. Jones GA, Butler J, Lieberman I, Schlenk R. Negativepressure wound therapy in the treatment of complex postoperative spinal wound infections: complications and lessons learned using vacuum-assisted closure. J Neurosurg Spine. 2007;6(5):407-411. doi:10.3171/spi.2007.6.5.407

7. Picada R, Winter RB, Lonstein JE, et al. Postoperative deep wound infection in adults after posterior lumbosacral spine fusion with instrumentation: incidence and management. $J$ Spinal Disord. 2000;13(1):42-45. doi:10.1097/00002517200002000-00009

8. Glassman SD, Dimar JR, Puno RM, Johnson JR. Salvage of instrumental lumbar fusions complicated by surgical wound infection. Spine (Phila Pa 1976). 1996;21(18):2163-2169. doi:10.1097/00007632-199609150-00021

9. Anthony S, Terrazas S. A retrospective study: clinical experience using vacuum-assisted closure in the treatment of wounds. J Natl Med Assoc. 2004;96(8):1073-1077.

10. Canavese F, Gupta S, Krajbich JI, Emara KM. Vacuumassisted closure for deep infection after spinal instrumentation for scoliosis. J Bone Joint Surg Br. 2008;90(3):377-381. doi:10. 1302/0301-620X.90B3.19890

11. Ploumis A, Mehbod AA, Dressel TD, Dykes DC, Transfeldt EE, Lonstein JE. Therapy of spinal wound infections using vacuum-assisted wound closure: risk factors leading to resistance to treatment. J Spinal Disord Tech. 2008;21(5):320 323. doi:10.1097/BSD.0b013e318141f99d

12. Morykwas MJ, Argenta LC, Shelton-Brown EI, McGuirt W. Vacuum-assisted closure: a new method for wound control and treatment: animal studies and basic foundation. Ann Plast Surg. 1997;38(6):553-562. doi:10.1097/ 00000637-199706000-00001

13. Labler L, Keel M, Trentz O, Heinzelmann M. Wound conditioning by vacuum assisted closure (V.A.C.) in postoperative infections after dorsal spine surgery. Eur Spine $J$. 2006;15(9):1388-1396. doi:10.1007/s00586-006-0164-2

14. Fox A, Tadros A, Perks AG. An unusual complication of vacuum assisted closure in the treatment of a pressure ulcer. $J$ Wound Care. 2004;13(8):344-345. doi:10.12968/jowc.2004.13. 8.26658

Disclosures and COI: This research received no specific grant from any funding agency in the public, commercial, or not-for-profit sector. This study received Exemption Category \#4, exempt from SUNY Upstate Medical University Institutional Review Board.

Corresponding Author: William F. Lavelle, MD, Department of Orthopaedic Surgery, SUNY 
Upstate Medical University, Syracuse, NY 13210. This manuscript is generously published free of Phone: (315) 464-8602; Fax: (315) 464-5223; Email: lavellew@upstate.edu.

Published 11 November 2020 charge by ISASS, the International Society for the Advancement of Spine Surgery. Copyright (C) 2020 ISASS. To see more or order reprints or permissions, see http://ijssurgery.com. 